\title{
Corona Onset Voltage at High Frequency for an Isolated, Cylindrical Electrode
}

\author{
William O. Price \\ The Boeing Company \\ Seattle, WA, USA \\ David V. Thiel \\ Griffith University \\ Queensland, Australia
}

\author{
John Drapala \\ The Boeing Company \\ Seattle, WA, USA
}

\author{
Robert G. Olsen \\ Washington State University \\ Pullman, WA, USA
}

\begin{abstract}
Corona on antennas or transmission lines is a significant source of electromagnetic interference on aircraft. A corona onset criterion, which describes breakdown in nonuniform RF fields, is derived from the Boltzmann transport equation. Predictions of corona onset are made using recent formulae for net ionization. Measurements of $300 \mathrm{MHz}$ corona onset for an isolated, cylindrical electrode were made and are compared with predictions. The results confirm that the derived breakdown criterion is a good predictor of RF corona onset at $300 \mathrm{MHz}$ for cylindrical geometries where diffusion is the dominant electron loss mechanism in the range $0.1 \leq r p \leq 10$ cm-torr.
\end{abstract}

Keywords—corona onset; high frequency breakdown

\section{INTRODUCTION}

After the British invention of the cavity magnetron in 1940 came a series of developments on both sides of the Atlantic that increased the power and efficiency of microwave devices by orders of magnitude [1]. It soon became apparent that a limit to further development was the electrical breakdown of air in cavities and transmission lines. In 1948 Herlin and Brown [2-4] published a diffusion theory of high frequency gas discharge breakdown that accurately predicted breakdown in coaxial structures and cylindrical cavities.

As the jet age dawned and high-power communications and sensors found their way onto high-altitude air vehicles a new problem arose: the breakdown of air around antennas and open transmission lines. The onset of corona in highpower RF systems often resulted in loss of function, interference to other systems and damage. Scientists in government and industry soon applied the theory of electron diffusion to this new problem. Most notable were the efforts of Chown, Morita, Scharfman and Taylor [5-10]; Fante, Yos and Otazo [11-15]; and Woo [16].

A previous work derived a criterion for corona onset (the Townsend integral) at $60 \mathrm{~Hz}$ for simple cylindrical geometries from the Boltzmann transport equation and showed good agreement with experimental results [17]. The first goal of this work was to derive a criterion for high frequency breakdown for simple cylindrical geometries from the Boltzmann transport equation. A second goal was to predict the corona onset voltage for a simple cylindrical geometry at $300 \mathrm{MHz}$ using expressions for $\alpha$ and $\eta$ developed by Schroeder et al. [18]. A third goal was to describe measurements of the corona onset voltage for a hemispherically capped monopole at $300 \mathrm{MHz}$ and compare with the predictions.

\section{Corona Onset Prediction}

Predictions of corona onset were made using a criterion for high frequency breakdown derived from the Boltzmann transport equation (see Appendix) and equations by Schroeder [19] fitted to ionization data compiled by Badaloni and Gallimberti [20]:

$$
\begin{aligned}
& \frac{\eta}{p}=1.95 \frac{\exp (-60 / x)}{x} \\
& \frac{\alpha}{p}=\left\{\begin{array}{l}
\exp \left(\frac{x-5.82 \times 10^{1}}{4.95}\right) \quad x \leq 35 \\
2.24 \times 10^{-3} x-6.92 \times 10^{-2} \quad x \leq 45 \\
-3.207 \times 10^{-12} x^{5}+3.259 \times 10^{-9} x^{4}-1.485 \times 10^{-6} x^{3}+ \\
3.44 \times 10^{-4} x^{2}-1.875 \times 10^{-2} x+3.013 \times 10^{-1} \quad x \leq 250 \\
1.45 \times 10^{1} \exp \left(\frac{-3.56 \times 10^{2}}{x}\right) \quad x>250,
\end{array}\right.
\end{aligned}
$$

where $x=E / p$ in $\mathrm{V} / \mathrm{cm}$-torr and the attachment and ionization coefficients are expressed in units of $1 / \mathrm{cm}$-torr. Schroeder's formulae were chosen because they describe net ionization over a range of $E / p$ greater than those of Sarma and Janischewskyj [21] (see fig. 1). 


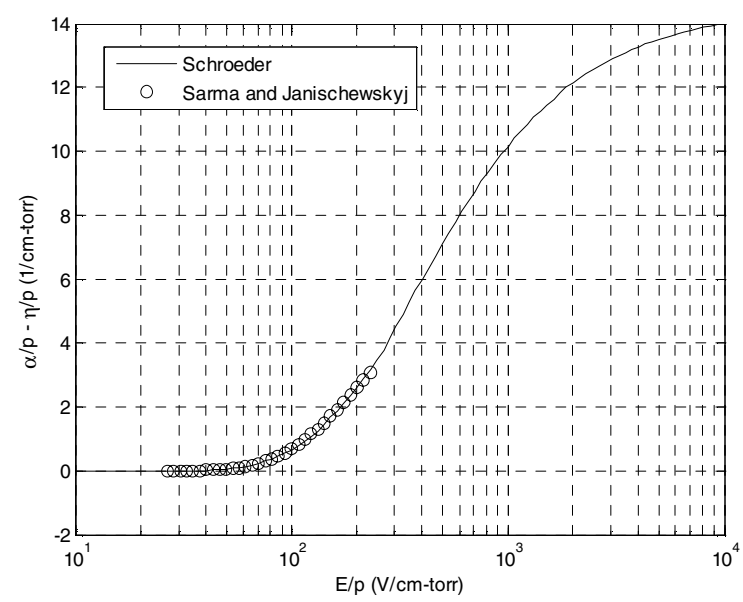

Figure 1. Net Ionization Formulae Compared

\section{MEASUREMENT OF CORONA OnSET}

Corona onset voltages were measured for a hemispherically capped monopole in a vacuum chamber as a function of pressure and temperature.

The monopole was 0.25 meters long with a radius of $3.8 \times 10^{-4}$ meters. It was manufactured of stainless steel and the hemispherical cap was shaped using microscopic inspection. The monopole was mounted on a ground plane 0.7 meters square that served as the floor of the vacuum chamber. Paraffin was molded on the lower $5 \mathrm{~cm}$ of the monopole to prevent breakdown between the monopole and the ground plane.

The vacuum chamber was completed with a glass bell. The bell was 0.78 meters tall at the center of the dome and 0.23 meters in radius. The monopole was mounted in the center of the bell's interior on the vacuum plate.

The monopole was excited with a signal source and RF amplifier at $300 \mathrm{MHz}$ (see Fig. 2). Ultraviolet illumination from a mercury vapor arc promoted electron production at the surface of the antenna.

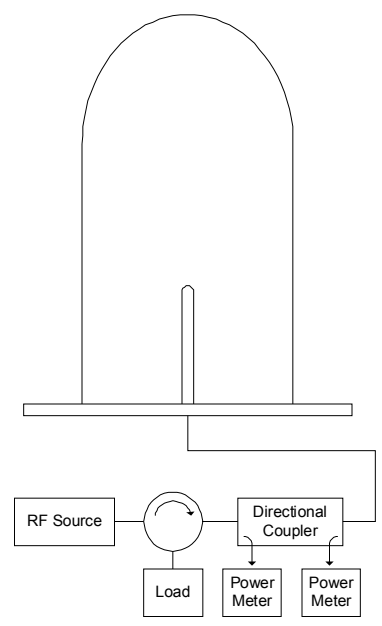

Figure 2. Experimental Apparatus
Before a series of measurements were made, the bell, ground plane and monopole were carefully cleaned. The monopole was repeatedly cleaned between corona events. In preparation for a measurement the chamber was sealed and pumped down to about 1 torr then backfilled with air dried through a desiccant. The chamber was evacuated to the target pressure and stabilized. Thus the air was entirely changed between each measurement of corona onset. Both temperature and pressure in the chamber were recorded.

The RF excitation was increased slowly until corona was noted. With the aid of the ultraviolet source corona was established in much less than a second upon reaching the corona onset voltage.

Corona onset and extinction were detected using the unaided human eye per ANSI Standard C29.1 [22]. The chamber was located in a darkened, shielded room and the observer's eyes were allowed to adjust to the darkness before attempting measurements. Corona inception was consistently observed at the tip of the monopole.

Measurements were made at 14 pressures between 5 and 100 torr. Results are plotted in Figure 3.

\section{Numerical Analysis of Surface Electric Field}

An analysis of the monopole and ground plane was performed using the Numerical Electromagnetic Code (NEC) [23] to correlate the antenna current distribution with the electric field intensity at the surface of the monopole. The electric field at the surface of the monopole is dominated by the quasistatic component [24] and is calculated either from an estimate of the antenna's charge distribution or by extrapolating the calculated near electric field to the surface assuming $\frac{1}{r}$ dependence [8]. The ratio of electric field intensity at the tip of the monopole to the drive point current was calculated to be $2180 \mathrm{ohm} / \mathrm{cm}$ at $300 \mathrm{MHz}$.

\section{RESUlTS}

Fig. 3 compares predictions of corona onset with measurements. The data is presented in proper variables, i.e. $E / p$ in $\mathrm{V} / \mathrm{cm}$-torr as a function of $r p$ in $\mathrm{cm}$-torr where $E$ is electric field intensity, $p$ is pressure and $r$ is the radius of the electrode.

Pressure was corrected for temperature using the formula,

$$
p=p_{\text {observed }}\left(\frac{273}{273+T}\right)
$$

where $T$ is temperature in degrees Centigrade.

The RF corona onset criterion predicted by (20) can be fitted to a $2^{\text {nd }}$ order polynomial curve valid over the range $0.1 \leq r p \leq 100 \mathrm{~cm}$-torr with a correlation coefficient

$R^{2}=0.988$,

$$
\begin{aligned}
& y=0.0588 x^{2}-0.449 x+4.44 \\
& x=\ln \left(r_{0} p\right) \text { cm-torr } \\
& y=\ln \left(E_{0} / p\right) \text { volts/cm-torr }
\end{aligned}
$$


The results confirm that at high frequency, the electron continuity equation with only the diffusion loss term is a good predictor of corona onset for isolated cylindrical geometries over the range $0.1 \leq r p \leq 100 \mathrm{~cm}$-torr.

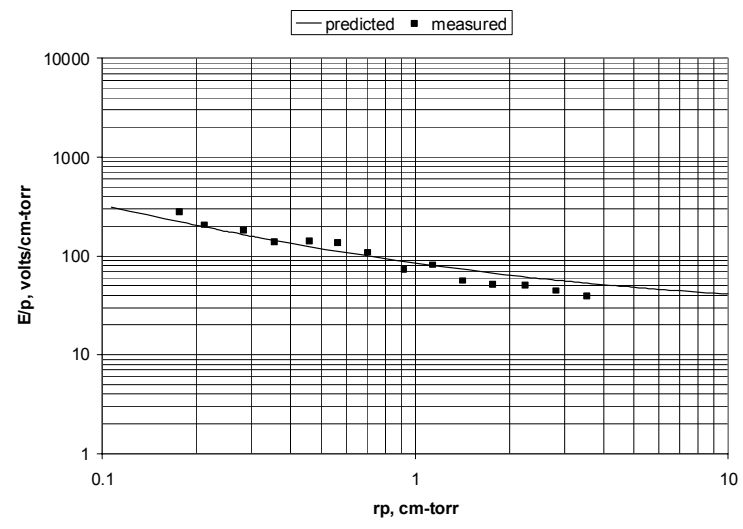

Figure 3. Predicted and Measured Corona Onset

\section{APPENDIX: HIGH FREQUENCY BREAKDOWN CRITERION}

In an appendix to his 1965 paper on microwave breakdown, Fante [11] derived the electron continuity equation from the Boltzmann transport equation,

$$
\frac{\partial f}{\partial t}+\nabla_{r} \cdot \mathbf{v} f+\nabla_{\mathrm{v}} \cdot \mathbf{a} f=J
$$

where $f(\mathbf{r}, \mathbf{v}, t)$ is the electron probability density function ${ }^{1}, \mathbf{r}$ is position, $\mathbf{v}$ is electron velocity, $\mathbf{a}$ the acceleration due to force and $J$ is the "binary collision integral" or, in this case, the supply of electrons due to ionizing collisions. The Boltzmann equation governs behavior in six dimensions, three in configuration space and three in velocity space.

If both sides of (4) are multiplied by an arbitrary function, $Q(\mathrm{v})$, and integrated over velocity, $Q=1$ yields

$$
\frac{\partial n}{\partial t}=-\nabla \cdot \Gamma+\left(v_{i}-v_{a}\right) n
$$

where $n$ is electron density, $\Gamma$ is electron current density, $v_{i}$ and $v_{a}$ are ionization and attachment frequencies respectively. The electron current density requires definition and that is achieved by multiplying both sides of the Boltzmann equation by $Q(\mathrm{v})=\mathrm{v}$ and integrating over velocity. The result is

$$
-n \frac{\partial\langle\mathrm{v}\rangle}{\partial t}=\nabla \frac{n \kappa T_{e}}{m}-\frac{n}{m} \mathbf{F}+\frac{n e}{m} \sum_{k} \mu_{k}^{-1}\left[\langle\mathrm{v}\rangle-\left\langle V_{k}\right\rangle\right](6)
$$

Where $T_{e}$ is electron temperature, $\mathbf{F}$ is force, $\mu_{k}$ is the mobility of the $k^{\text {th }}$ neutral species and $\left\langle V_{k}\right\rangle$ is the ensemble averaged velocity of the $k^{\text {th }}$ neutral species. In the steady state equation (6) yields for $\Gamma=n\langle\mathrm{v}\rangle$ (assuming all species

${ }^{1} f(\mathbf{x}, \mathbf{v}, t) d^{3} x d^{3} \mathbf{v}$ is defined as the number of electrons in a volume $d^{3} x d^{3} \mathrm{v}$ about $(\mathbf{x}, \mathbf{v})$ at time $t$. have the same velocity $V$ and $\mu_{k}$ is independent of spatial coordinates)

$$
n\langle\mathrm{v}\rangle=n\langle V\rangle+\frac{n}{e} \mu \mathbf{F}-\nabla D n
$$

Where $V$ is the velocity of convective flow, $\mathbf{F}$ is the timeaveraged force due to the electrical field, $D$ is the diffusion coefficient and $\mu$ is electron mobility.

$$
\begin{aligned}
& D=\frac{\mu \kappa T_{e}}{e} \\
& \mu=\frac{1}{\sum_{k} \frac{1}{\mu_{k}}}
\end{aligned}
$$

When force is stated in terms of electric field intensity

$$
\Gamma=n\langle\mathbf{V}\rangle-n \mu \mathbf{E}-\nabla D n .
$$

The three terms on the right-hand side of (9) are, in order, convection,' drift and diffusion. Herlin and Brown [3] state that in a microwave discharge, in which the excitation frequency is relatively large, drift is oscillatory and does not contribute to electron transport. Thus, if convection can be neglected, diffusion will be the only significant loss mechanism at high frequency. ${ }^{2}$

When electron diffusion is the dominant loss mechanism, then

and (5) becomes

$$
\Gamma=-\nabla D n
$$

$$
\frac{\partial n}{\partial t}=\nabla^{2} D n+v n
$$

where $v$ is the net ionization frequency. Since the diffusion coefficient, $D$, varies slowly as a function of electric field intensity, $D \nabla^{2} n \gg n \nabla^{2} D$ and so

$$
\frac{\partial n}{\partial t} \simeq D \nabla^{2} n+v n
$$

If we assume

$$
n(r, t)=\theta(r) \phi(t)
$$

then the breakdown condition is described in cylindrical coordinates by the homogeneous ordinary differential equation [3]

$$
\theta^{\prime \prime}+\frac{1}{r} \theta^{\prime}+\frac{v}{D} \theta=0
$$

which is Bessel's equation and has Bessel functions of integer order for solutions.

With application of appropriate boundary conditions it is possible, in principle, to derive a non-trivial solution by use of Cramer's rule. In several papers by Herlin and Brown [24] breakdown is calculated between coaxial cylinders. The walls of the inner and outer cylinders are natural boundaries where the electron density, $n$, vanishes. Fante et al. [14] derived similar breakdown conditions for coaxial cylinders but with the provision that the distance between them is so great that an ionization boundary may form (where $\left.v=v_{i}-v_{a}=0\right)$. In such a case there are three natural boundaries: the two cylinder walls and the ionization

\footnotetext{
${ }^{2}$ An example of convection would be airflow.
} 
boundary where there is a singularity. Fante's approach was to form two problems with three boundaries. In the interior region where $v>0, v \simeq v_{i}$; in the outer region where $v<0, v \simeq v_{a}$. At the cylinder walls the electron density vanishes and at the ionization boundary the solutions and their first derivatives are set equal to each other. By this means Fante calculated breakdown criteria in good agreement with experimental results. As the outer boundary becomes very large or infinite, the solution describes corona onset for an isolated electrode.

If one uses proper variables, i.e. $E / p$ in $\mathrm{V} / \mathrm{cm}$-torr as a function of $\rho=r p$ in cm-torr where $E$ is electric field intensity, $p$ is pressure and $r$ is radius and, further, if $\rho_{0}$ describes the surface of the cylindrical electrode, $\rho_{1}$ the ionization boundary and $\rho_{2}$ an arbitrarily large outer boundary at which the electron density is vanishingly small then in the ionization region $\left(\rho_{1} \leq \rho \leq \rho_{2}\right)$ electron density is described as

$$
\frac{\partial^{2} n}{\partial \rho^{2}}+\frac{1}{\rho} \frac{\partial n}{\partial \rho}+\frac{v_{i}}{D} v=0
$$

And the solution is

$$
n_{1}(\rho)=a_{1} J_{0}\left(\lambda_{1} \rho\right)+a_{2} Y_{0}\left(\lambda_{1} \rho\right)
$$

where $\lambda_{1}=\sqrt{\frac{\nu_{i}}{D}}$.

In the attachment region $\left(\rho>\rho_{1}\right)$ the expression for electron density is

$$
\frac{\partial^{2} n}{\partial \rho^{2}}+\frac{1}{\rho} \frac{\partial n}{\partial \rho}-\frac{v_{a}}{D} n=0
$$

And the solution is

$$
n_{2}(\rho)=a_{3} K_{0}\left(\lambda_{2} \rho\right)+a_{4} I_{0}\left(\lambda_{2} \rho\right)
$$

where $\lambda_{2}=\sqrt{\frac{\nu_{a}}{D}}$.

The applicable boundary conditions are

$$
\begin{aligned}
& n_{1}\left(\rho_{0}\right)=0, \\
& n_{2}\left(\rho_{2}\right)=0 \text { as } \rho_{2} \rightarrow \infty, \\
& n_{1}\left(\rho_{1}\right)=n_{2}\left(\rho_{1}\right) \text { and } \\
& n_{1}^{\prime}\left(\rho_{1}\right)=n_{2}^{\prime}\left(\rho_{1}\right)
\end{aligned}
$$

Solutions exist for values of $\lambda_{1}$ and $\lambda_{2}$ for which

$$
\left|\begin{array}{cccc}
J_{0}\left(\lambda_{1} \rho_{0}\right) & Y_{0}\left(\lambda_{1} \rho_{0}\right) & 0 & 0 \\
0 & 0 & K_{0}\left(\lambda_{2} \rho_{2}\right) & I_{0}\left(\lambda_{2} \rho_{2}\right) \\
J_{0}\left(\lambda_{1} \rho_{1}\right) & Y_{0}\left(\lambda_{1} \rho_{1}\right) & -K_{0}\left(\lambda_{2} \rho_{1}\right) & -I_{0}\left(\lambda_{2} \rho_{1}\right) \\
J_{0}^{\prime}\left(\lambda_{1} \rho_{1}\right) & Y_{0}^{\prime}\left(\lambda_{1} \rho_{1}\right) & -K_{0}^{\prime}\left(\lambda_{2} \rho_{1}\right) & -I_{0}^{\prime}\left(\lambda_{2} \rho_{1}\right)
\end{array}\right|=0
$$

From (20) it is possible to calculate electric field strength limits for corona onset for a particular pressure and electrode radius.

[1] R. Buderi, The Invention that Changed the World. New York: Simon \& Schuster, Inc., 1996.
[2] M. A. Herlin and S. C. Brown, "Electrical Breakdown of a Gas between Coaxial Cylinders at Microwave Frequencies," Physical Review, vol. 74, pp. 910-913, 1948.

[3] M. A. Herlin and S. C. Brown, "Breakdown of a Gas at Microwave Frequencies," Physical Review, vol. 74, pp. 291296, 1948.

[4] M. A. Herlin and S. C. Brown, "Microwave Breakdown of a Gas in a Cylindrical Cavity of Arbitrary Length," Physical Review, vol. 74, pp. 1650-1656, 1948

[5] J. Chown, T. Morita, and W. Scharfman, "Voltage breakdown characteristics of microwave antennas," presented at IRE International Convention Record, 1958.

[6] J. M. Chown, T.; Scharfman, W., "Voltage Breakdown Characteristics of Microwave Antennas," Proceedings of the IRE, pp. 1331-1337, 1959.

[7] W. Scharfman and T. Morita, "Power-handling capability of antennas at high altitude," presented at IRE International Convention Record, 1960

[8] W. Scharfman and T. Morita, "Voltage Breakdown of Antennas at High Altitudes," Stanford Research Institute, Menlo Park Technical Report 69, April 1960.

[9] W. Scharfman, W. C. Taylor, and T. Morita, "Research Study of Microwave Breakdown of Air at High Altitudes," Air Force Cambridge Research Laboratories, Bedford, MA August 1962 1962.

[10] W. C. Taylor, W. Scharfman, and T. Morita, "Voltage Breakdown of Microwave Antennas," in Advances in Microwaves, vol. 7, L. Young, Ed. New York: Academic Press, 1971, pp. 59-130.

[11] R. Fante, "Mathematical analysis of microwave breakdown in flowing gases," Antennas and Propagation, IEEE Transactions on [legacy, pre - 1988], vol. 13, pp. 781-788, 1965.

[12] R. M. Fante, J., "Bounds on the electric field outside a radiating system," Antennas and Propagation, IEEE Transactions on [legacy, pre - 1988], vol. 16, pp. 712-717, 1968.

[13] R. M. Fante, J., "Bounds on the electric field outside a radiating system--II," Antennas and Propagation, IEEE Transactions on [legacy, pre - 1988], vol. 18, pp. 64-68, 1970.

[14] R. L. Fante, J. M. Yos, and J. J. Otazo, "Simple Analytical Models for Calculating Breakdown in Air-Filled Transmission Systems," Air Force Cambridge Research Laboratories AFCRL-71-0206, April 1, 19711971.

[15] J. M. Yos, "Microwave Breakdown Predictions for a Rectangular Aperture Antenna Including Lateral Diffusion," Air Force Cambridge Research Laboratories, Bedford, MA AFCRL-71-0120, February 18, 19711971.

[16] R. Woo, "Final Report on RF Voltage Breakdown in Coaxial Transmission Lines," Jet Propulsion Laboratory, Pasadena, CA Technical Report 32-1500, October 1, 19701970.

[17] W. O. Price, D. V. Thiel, J. Drapala, and R. G. Olsen, "Corona onset voltage at 60 hertz for an isolated, cylindrical electrode," presented at 2006 IEEE International Symposium on Electromagnetic Compatibility, 2006.

[18] V. Schroeder, M. B. Baker, and J. Latham, "A model study of corona emission from hydrometeors," Quarterly Journal of the Royal Meteorological Society, vol. 125, pp. 1681-1693, 1998.

[19] V. Schroeder, "MATLAB functions of ionization and attachment coefficients." Seattle, 2000.

[20] S. Badaloni and I. Gallimberti, "The inception mechanism of the first corona in non-uniform gaps," University of Padua, Padua 72/05, 19721972.

[21] M. P. Sarma and W. Janischewskyj, "D.C. corona on smooth conductors in air," Proceedings of the IEE, vol. 116, pp. 161166,1969 .

[22] "ANSI Standard C29.1--1988, for Electrical Power Insulators-Test Methods," American National Standards Institute, New York August 23, 19981988.

[23] G. A. Burke and A. J. Poggio, "Numerical Electromagnetics Code (NEC)," 2 ed. Livermore: Lawrence Livermore Laboratory, 1981.

[24] S. A. Schelkunoff and H. T. Friis, Antennas, Theory and Practice. New York: John Wiley \& Sons, Inc., 1966. 